\title{
Effect of Percutaneous Radiofrequency Ablation and Surgical Resection on Treating of Primary Small Liver Cancer: A Meta-Analysis
}

Qian $\mathrm{He}^{1}$, Xingwu Yang ${ }^{2 *}$, Qi Wang ${ }^{2}$, Qian Wang ${ }^{2}$

${ }^{1}$ Shaanxi University of Traditional Chinese Medicine, Xianyang, Shaanxi Province, China;

${ }^{2}$ Affiliated Hospital of Shaanxi University of Traditional Chinese Medicine, Hepatological Surgery, Xianyang, Shaanxi Province, China

Abstract: Objective: To analyze the clinical effect of primary small liver cancer under radiofrequency ablation (RFA) and surgical resection (SR) treatment, and to explore the best application timing of the two treatment methods. Methods: Randomly controlled clinical trials (RCT) of percutaneous radiofrequency ablation and surgical resection for the treatment of small liver cancer in various databases were searched according to the established search strategy, and Meta data was analyzed based on RevMan5.3 software provided by Cochrane collaborative organization. Results: A total of 10 articles were included in this study, including 1396 patients, 699 in the RFA group and 697 in the SR group. Meta-analysis results showed that the two groups had no statistical difference in overall survival rate at 1 and 3 years, 1 -year diseasefree survival rate, and 1-year recurrence rate $(P>0.05)$. The 3-year disease-free survival rate of the RFA group was lower than that of the SR group, and the 3 -year recurrence rate was higher than that of the SR group. The higher postoperative complication rate tended to the SR group, and the differences were statistically significant. $(P<0.05)$. Conclusion: For short-term efficacy, radiofrequency ablation and surgical resection show consistency; for long-term efficacy, surgical resection is more effective.

Key words: Primary liver cancer; Radiofrequency ablation; Surgical resection; Randomized controlled study; Meta analysis

Publication date: January, 2021
Publication online: 31 January, 2021

"Corresponding author: Xingwu Yang, heqian1128@ 163.com

At present, primary liver cancer occupies the fourth place in the incidence of gastrointestinal tumors in China, and ranks second in tumor-related mortality. The main morbidity factors are viral hepatitis, aflatoxin-contaminated food, long-term smoking, alcoholism, type 2 diabetes, Obesity and other related $^{[1,2]}$. Studies have shown that the overall survival rate of primary liver cancer has not changed significantly in the past decade, and its incidence is expected to continue to increase in the next few decades $^{[3]}$. The proportion of patients with clinical symptoms in the period of small liver cancer is too low. When symptoms such as fatigue, anorexia, abdominal distension, liver pain, and jaundice appear, most of the cancer has advanced to the middle and late stages, and the quality of survival is almost lost. At this time, early diagnosis is particularly important. In recent years, with the improvement of diagnosis and treatment technology, the popularization of disease science, and the screening of high-risk populations, the early detection rate of diseases has increased, and the limited survival period of patients has the opportunity to be extended. Surgical resection, ablation and liver transplantation are the three recognized methods for the treatment of small liver cancer. Due to insufficient liver sources and economic factors, it means that only a very small number of patients benefit from liver transplantation. For the 
choice between the two, which treatment is effective the best, can strive for a longer survival period and a good quality of life for patients, there are still some controversies in actual clinical applications. The purpose of this study is to conduct a meta-analysis of randomized controlled studies on radiofrequency ablation and surgical resection for the treatment of primary small liver cancer, in order to find a more appropriate time for the clinical application of radiofrequency ablation and surgical resection.

\section{Materials and Methods}

\subsection{Literature search strategy}

Computer-assisted manual search of PubMed, Embase, The Cochrane Library, CBM, CNKI, Weipu Journal, Wanfang database, comprehensively collected randomized controlled studies on RFA and SR treatment of primary small liver cancer (2010.012020.02). Using a combination of subject terms and free words, search terms include primary liver cancer, liver tumors, small liver cancer, radiofrequency ablation, and surgical resection. The search languages are mainly English and Chinese, excluding abstracts and reports that have not yet been published.

\subsection{Literature inclusion and exclusion criteria}

\subsubsection{Type of Study}

A randomized controlled clinical study of radiofrequency ablation and surgical resection for the treatment of primary small liver cancer regardless of the sample size, whether blinding and allocation concealment are used.

\subsubsection{Include objects}

The patient's first diagnosis was primary liver cancer; Single lesion smaller than $5 \mathrm{~cm}$ or less than 3 multiple lesions smaller than $3 \mathrm{~cm}$; Limited to liver function at Child-Pugh A or B level; No extrahepatic metastasis and peripheral vascular invasion; First anti-cancer treatment; Both belong to the scope of indications for surgical resection or radiofrequency ablation, and there are no contraindications for both treatment methods.

\subsubsection{Intervention}

The experimental group received radiofrequency ablation (RFA) therapy, and the control group received surgical resection (SR) therapy.

\subsubsection{Outcome indicators}

1, 3-year overall survival, disease-free survival rate, recurrence rate and postoperative complications rate of patients

\subsubsection{Exclusion criteria}

Patients with recurrent liver cancer or metastatic cancer; Cooperate with other treatment methods such as hepatic artery chemoembolization (TACE), stereotactic radiation, drug therapy, etc.; Non-firsttime anti-cancer patients; Those who are not classified as A or B liver function;Literatures where the main outcome indicators are too few or data cannot be extracted.

\subsection{Data Extraction}

Firstly, the initial screening is carried out by the title and abstract of the literature; then, based on the inclusion and exclusion criteria, carefully check whether to be included. Two researchers began to screen independently and cross-check the results.

Inconsistent or disputed parts of this result can be resolved through discussion or third-party assistance. Extract the first author, publication year, research methods, intervention measures, outcome indicators, follow-up and other basic information of the detected literature.

\subsection{Statistical analysis}

Relying on the Cochrane collaboration organization to provide RevMan5.3 software to complete the Meta analysis of the data. The odds ratio (OR) and its $95 \%$ $\mathrm{CI}$ are used to describe the count data, and the chisquare test is used to achieve heterogeneity detection between studies. When $I^{2}<50 \%$ and $P>0.05$, it means that there is no significant heterogeneity, The fixed-effects model can be used for Meta analysis; when $I^{2} \geq 50 \%$ and $P \leq 0.05$, it indicates that there is a certain degree of heterogeneity, then the randomeffects model can be used for analysis. $P<0.05$ means that the difference is statistically significant.

\section{Research result}

\subsection{Basic characteristics of search results and selected articles}

Based on the established search strategy, 1,213 articles were retrieved for the first time, and 626 articles were selected by reading the titles and abstracts of the articles. Then follow the established 
inclusion and exclusion criteria to read the full text intensively, and finally selected 10 articles, a total of 1396 patients, including 699 cases in the RAF group and 697 cases in the SR group, as shown in Figure 1. In the included 10 studies, the basic conditions of the two groups of patients, such as average age, sex ratio, tumor size, etc., were not statistically different, and the baselines between the groups were basically the same. See Table 1 for details.

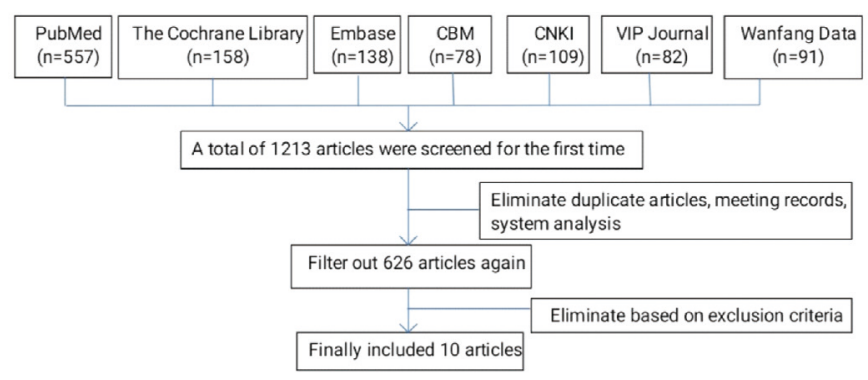

Figure 1. Flow chart of article screening

Table 1. Basic information of included articles

\begin{tabular}{|c|c|c|c|c|c|c|c|}
\hline $\begin{array}{l}\text { Include } \\
\text { articles }\end{array}$ & $\begin{array}{c}\text { Random } \\
\text { approach to } \\
\text { experimental } \\
\text { design }\end{array}$ & Group & $\begin{array}{l}\text { Number } \\
\text { of cases }\end{array}$ & $\begin{array}{l}\text { Average age } \\
\text { (years) }\end{array}$ & $\begin{array}{l}\text { Male/female } \\
\text { (example) }\end{array}$ & $\begin{array}{c}\text { Follow-up } \\
\text { time } \\
\text { (months) }\end{array}$ & $\begin{array}{l}\text { Article } \\
\text { quality } \\
\text { score }\end{array}$ \\
\hline \multirow[t]{2}{*}{ Zhong et al. (2015) ${ }^{[4]}$} & Lottery & RFA & 40 & $61.58 \pm 10.87$ & $19 / 21$ & 24 & 3 \\
\hline & & SR & 38 & $62.67 \pm 9.67$ & $20 / 18$ & & \\
\hline \multirow[t]{2}{*}{ Bai et al. $(2020)^{[5]}$} & Random number table & RFA & 50 & $57 \pm 4$ & $29 / 21$ & 36 & 3 \\
\hline & & SR & 50 & $57 \pm 4$ & $31 / 19$ & & \\
\hline \multirow[t]{2}{*}{ Ding et al. $(2017)^{[6]}$} & Random number method & RFA & 54 & $57.72 \pm 10.63$ & $34 / 20$ & 36 & 4 \\
\hline & & SR & 54 & $58.14 \pm 10.83$ & $35 / 19$ & & \\
\hline \multirow[t]{2}{*}{ Lian et al. (2016) } & Not specified & RFA & 63 & $56.83 \pm 6.62$ & $40 / 23$ & 36 & 2 \\
\hline & & SR & 63 & $57.09 \pm 5.84$ & $43 / 20$ & & \\
\hline \multirow[t]{2}{*}{ Li et al. $(2020)^{[8]}$} & Not specified & RFA & 100 & $58.12 \pm 2.33$ & $100 / 90$ & 36 & 2 \\
\hline & & SR & 100 & & & & \\
\hline \multirow[t]{2}{*}{ Cui et al. (2018) } & Random number table & RFA & 42 & $64.39 \pm 3.41$ & $29 / 13$ & 12 & 4 \\
\hline & & SR & 42 & $64.31 \pm 3.45$ & $30 / 12$ & & \\
\hline \multirow[t]{2}{*}{ Bai et al. $(2014)^{[10]}$} & Not specified & RFA & 42 & $47.2 \pm 5.8$ & $22 / 20$ & 36 & 3 \\
\hline & & SR & 42 & $52.3 \pm 6.6$ & $23 / 19$ & & \\
\hline \multirow[t]{2}{*}{ Huang et al.(2010) ${ }^{[11]}$} & $\begin{array}{l}\text { Computer blocking/layered randomization } \\
\text { method }\end{array}$ & RFA & 115 & $56.6 \pm 14.3$ & $79 / 36$ & 37.2 & 5 \\
\hline & & SR & 115 & $55.9 \pm 12.6$ & $85 / 30$ & 46.4 & \\
\hline \multirow[t]{2}{*}{ Feng et al.(2012) ${ }^{[12]}$} & Computer random number generator & RFA & 84 & $47(18-76)$ & $79 / 5$ & 36 & 5 \\
\hline & & SR & 84 & $51(24-83)$ & $75 / 9$ & & \\
\hline \multirow[t]{2}{*}{ Ng et al. $(2017)^{[13]}$} & $\begin{array}{l}\text { Random grouping of consecutively } \\
\text { numbered sealed envelopes }\end{array}$ & RFA & 109 & $57(23-78)$ & $86 / 23$ & 93 & 5 \\
\hline & & SR & 109 & $55(31-82)$ & $89 / 20$ & & \\
\hline
\end{tabular}

Note: RFA: Radiofrequency ablation group SR: Surgical resection group

\subsection{Article quality evaluation}

The improved version of the Jadad scale was used to complete the quality assessment of the included studies. This study included 10 articles, 5 of which were high quality (4-7 points) and 5 were low quality (1-3 points).

\subsection{Analysis of research results}

\subsubsection{1, 3-year overall survival analysis}

All of the included 10 articles reported the overall survival of patients at 1 year after surgery. All the articles were included in the analysis. A total of 1396 cases were included, including 699 cases in the RFA group and 697 cases in the SR group. There was no heterogeneity among the studies $\left(P=0.08, I^{2}=\right.$ $43 \%)$. After the analysis of the fixed effects model, the $O R=0.79$ ( $95 \% C I, 0.52$ to 1.19$), P=0.26$. The results show that there is no significant difference in the 1-year overall survival rate between RFA and SR treatment of primary small liver cancer(Figure 2). 


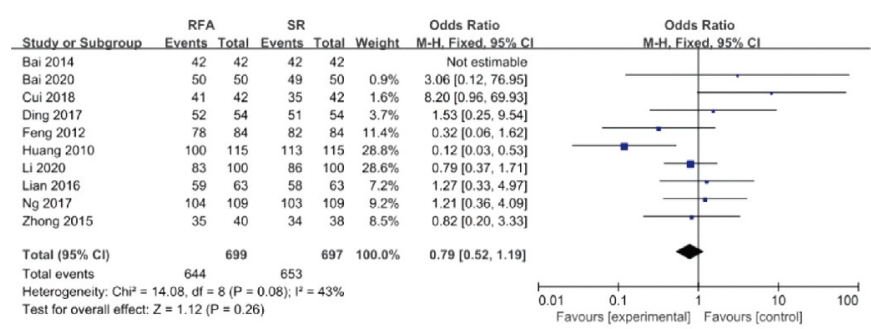

Figure 2. Comparison of 1-year overall survival rate

There are 8 articles describing the overall survival of patients at 3 years after surgery. The total number of cases studied was 1234, including 617 in the RFA group and 617 in the SR group. There was heterogeneity among the studies $\left(P=0.03, I^{2}=54 \%\right)$. After analysis with the random effects model, $O R=$ $0.72(95 \% C I, 0.49$ to 1.05$), P=0.09$. The analysis showed that there was no statistically significant difference in the 3-year overall survival rate between RFA and SR treatment of primary small liver cancer. (Figure 3)

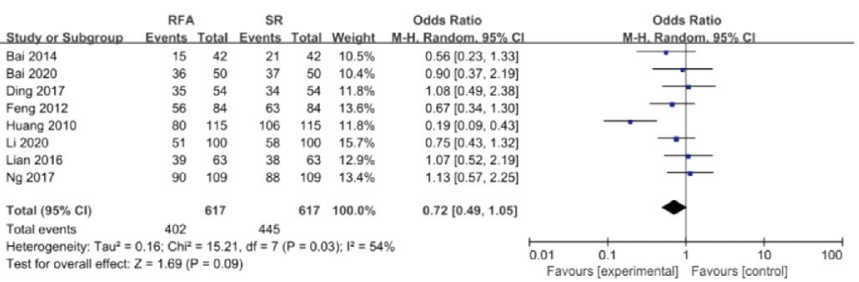

Figure 3. Comparison of 3-year overall survival rate

\subsubsection{1, 3-year disease-free survival analysis}

There are 5 articles on the analysis of patients' 1-year disease-free survival. The total number of cases in the study was 778, including 390 in the RFA group and 388 in the SR group. There is no significant heterogeneity among the studies $\left(P=0.98, I^{2}=0\right)$, using fixed-effects model analysis, that is, $O R=0.75$ (95\% $C I, 0.52$ to 1.09 ), $P=0.13$. It shows that the 1 -year disease-free survival rate of RFA and SR treatment of primary small liver cancer is similar. (Figure 4)

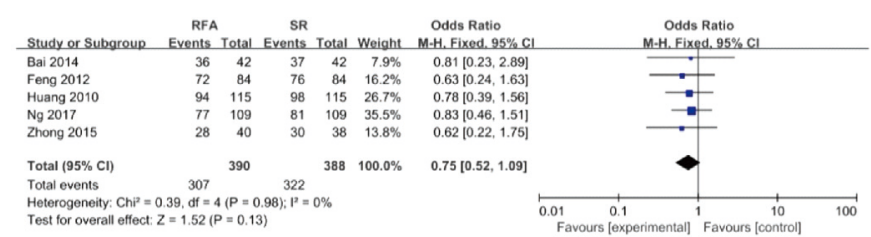

Figure 4. Comparison of 1-year disease-free survival rate

There are 4 studies that reported the disease-free survival of patients at 3 years after surgery. The total number of cases in the study was 700 , including 350 in the RFA group and 350 in the SR group. There is no heterogeneity among the studies $\left(P=0.68, I^{2}=0\right)$, and the analysis uses a fixed-effect model, that is, $O R$ $=0.69(95 \% C I, 0.51$ to 0.93$), P=0.01$, statistically significant. It shows that the 3-year disease-free survival rate of RFA treatment of primary small liver cancer is relatively low(Figure 5).

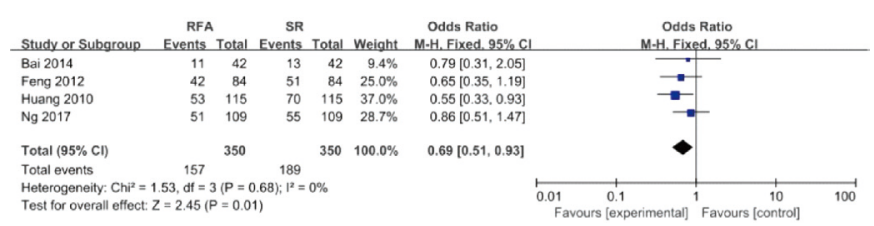

Figure 5. Comparison of 3-year disease-free survival rate

\subsubsection{1, 3-year recurrence rate analysis}

There are 6 articles describing the recurrence of patients one year after surgery. The total number of cases studied is 786, including 394 cases in the RFA group and 392 cases in the SR group. There was no heterogeneity among the studies ( $\left.P=0.09, I^{2}=48 \%\right)$. Using the random effects model, $O R=0.83(95 \%$ $C I, 0.57$ to 1.20$), \mathrm{P}=0.32$. It shows that there is no statistical significance in the one-year recurrence rate difference between RFA and SR treatment of primary small liver cancer(Figure 6).

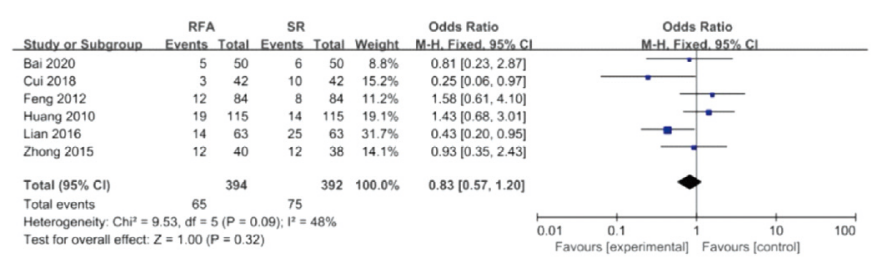

Figure 6. Comparison of 1-year recurrence rate

There are 6 articles that reported the recurrence of patients 3 years after surgery. The total number of cases in the study was 890 , including 445 cases in the RFA group and 445 cases in the SR group. There is no significant heterogeneity between studies $(P=0.75$, $\left.I^{2}=0\right)$. The analysis uses a fixed-effect model, which is $O R=1.60$ ( $95 \% C I, 1.21$ to 2.12 ), $P=0.009$, statistically significant. It shows that the 3 -year recurrence rate of RFA treatment of primary small liver cancer is relatively high(Figure 7).



Figure 7. Comparison of 3-year recurrence rate 


\subsubsection{Complication analysis}

There are 7 articles describing the occurrence of postoperative complications, with a total of 1066 cases, including 533 cases in the RFA group and 533 cases in the SR group. There is no significant heterogeneity among the studies $\left(P=0.26, I^{2}=22 \%\right)$. The analysis uses a fixed-effect model, which is OR $=0.234$ (95\% CI, 0.17 to 0.34$), P<0.00001$, the difference is statistically significant. It indicates that the postoperative complication rate of RFA treatment of primary small liver cancer is significantly lower (Figure 8).



Figure 8. Comparison of postoperative complications

\section{Discussion}

For the treatment of primary small liver cancer, liver transplantation is the most effective radical treatment, especially for patients with poor liver function who have recurred liver cancer after surgery. However, the lack of donors and the risks and costs of long-term immunosuppression after surgery have discouraged many patients and turned to other methods ${ }^{[14]}$. Surgery has a long history, and the continuous indepth understanding of the disease and the continuous advancement of imaging inspection technology, suitable surgical resection methods may also achieve a radical cure for some patients ${ }^{[15]}$. In recent years, the concept of "minimally invasive" has become more popular, and more and more patients tend to use minimally invasive treatment, believing that it has the advantages of less trauma, psychologically acceptable, fast recovery, and good treatment effects. But for the comparative analysis of the clinical efficacy of the two, the conclusion has not been consistent.

This study conducted a Meta analysis of randomized controlled clinical studies on surgical resection and radiofrequency ablation in the treatment of primary small liver cancer in the past ten years. The results showed that: in terms of overall survival rate, whether it is short-term or long-term, the two are not significant Difference; short-term diaease- free survival rate and recurrence rate, the two also show similarities; but for long-term disease-free survival rate and recurrence rate, surgical resection is still more dominant. The conclusions of this study are also consistent with the results of multiple retrospective analyses ${ }^{[16,17]}$. Of course, the benefits brought by the use of radiofrequency ablation cannot be ignored. It significantly reduces postoperative complications, such as infection, biliary fistula, blood loss, liver failure, etc.; it can also shorten the hospital stay and reduce economic costs; and it can be repeated mang times. How to grasp the application opportunity and make choices is the key. Jiang et al. ${ }^{[18]}$ studied the effect of SR and RFA on patients with very early or early liver cancer based on age and tumor size, and concluded that radiofrequency ablation is recommended for patients aged $>65$ years and tumors $<2 \mathrm{~cm}$; hepatectomy is a better choice for patients aged $>65$ years with tumors between $2-5 \mathrm{~cm}$ and $<65$ years with tumors of different sizes. Huang et al. ${ }^{[19]}$ analyzed the clinical efficacy of RFA and SR for liver cancer smaller than $2 \mathrm{~cm}$ and found that there was no significant difference between RFA and SR in overall survival. Ye et al. ${ }^{[20]}$ showed that for 3-4 cm single liver cancer, radiofrequency ablation and surgical resection have no significant difference in short-term and long-term prognosis, and have the advantages of low complication rate and low cost, while surgical resection is recommended for the long-term prognosis of a single liver cancer with a diameter of 4-5 cm, and tumors with a diameter of more than $4 \mathrm{~cm}$ are positively correlated with a high rate of satellite nodule formation and a high rate of microvascular invasion. Casadei et al. ${ }^{[21]}$ emphasized that in the case of single nodule tumors smaller than $2 \mathrm{~cm}$, Child-Pugh A liver function, albumin-bilirubin score 1, and alpha-fetoprotein lower than $20 \mathrm{ng} / \mathrm{mL}$, RFA has the best benefits in terms of overall survival and tumor-free survival. Pan et al. ${ }^{[22]}$ believed that the use of RFA for treatment of primary liver cancer less than $5 \mathrm{~cm}$ in the center of the liver can achieve similar results as surgical resection. Zheng et al. ${ }^{[23]}$ proved that SR has a positive effect on OS and CSS of patients with $3.1-5 \mathrm{~cm}$ tumors through effective sensitivity analysis; when the tumor size is less than 3 $\mathrm{cm}$, the survival benefits of patients from SR and RFA treatment are similar. Combined with the results of multiple studies, it can be seen that in the face of the choice of treatment for primary small liver cancer, not 
only the size of the liver cancer is considered, but the result of a comprehensive consideration of multiple factors. In the clinic, the optimal treatment plan suitable for the individual can be selected according to multiple factors such as tumor size, tumor location, liver function classification, and age.

This article also has many shortcomings. Since the tumor size selected by the included randomized controlled studies is less than $5 \mathrm{~cm}$, and no further subgroup analysis is made based on the tumor size, this article only makes an analysis for tumors less than $5 \mathrm{~cm}$; the quality assessment of the included literature shows that the overall level is low. It is expected that there will be a large number of prospective randomized controlled clinical studies with a large sample size to provide more powerful support for the selection of clinicians.

\section{References}

[1] Liu, Z., et al., Global incidence trends in primary liver cancer by age at diagnosis, sex, region, and etiology, 1990 - 2017[J]. Cancer, 2020. 126(10): 2267-2278

[2] Standards for diagnosis and treatment of primary liver cancer_2019 edition_JJ]. Chinese Journal of Clinical Medicine, 2020. 27(1):140

[3] Mejia, J.C. , J. Pasko, Primary Liver Cancers[J].Surgical Clinics of North America, 2020. 100(3): 535-549

[4] Zhong Yongwei, Luo Jiankun, Wu Yongqiang. Comparison of the efficacy of radiofrequency ablation therapy and surgical resection for primary small liver cancer[J]. Shenzhen Journal of Integrated Traditional Chinese and Western Medicine, 2015. 25(14):97-99

[5] Bai Jing et al., Clinical study of ultrasound-guided percutaneous radiofrequency ablation in the treatment of primary hepatocellular carcinoma[J]. China Medicine, 2020. 15(01): 71-74

[6] Ding Tao ,Wei Jian. Comparison of short-term and long-term efficacy and safety of percutaneous radiofrequency ablation and surgical resection for small liver cancer patients[J]. Chinese Journal of Integrated Traditional Chinese and Western Medicine on Digestion, 2017. 25(12): 929-932

[7] Lian Yuanshu, Yang Hao. Comparison of clinical effects of percutaneous radiofrequency ablation and surgical resection for small liver cancer[J]. Clinical Medical Research and Practice, 2016. 1(06): 16-18

[8] Li Jingfeng, Qiao Juan. Analysis of the effect of radiofrequency ablation and hepatocarcinectomy in the treatment of primary small liver cancer and the occurrence of complications $[\mathrm{J}]$.
Mental Monthly, 2020. 15(04): 191

[9] Cui Faqiang, Li Tao, Yin Tianying. The effect of radiofrequency ablation in the treatment of primary small liver cancer[J]. Clinical Medical Research and Practice, 2018. 3(36): $56-58$

[10] Bai Chunhui et al., Comparative study of radiofrequency ablation and resection in the treatment of small liver cancer[J]. Modern Journal of Integrated Traditional Chinese and Western Medicine, 2014. 23(36): 4042-4043

[11] Huang, J., et al., A Randomized Trial Comparing Radiofrequency Ablation and Surgical Resection for HCC Conforming to the Milan Criteria[J].Annals of Surgery, 2010. 252(6): 903-912

[12] Feng, K., et al., A randomized controlled trial of radiofrequency ablation and surgical resection in the treatment of small hepatocellular carcinoma[J]. Journal of Hepatology, 2012. 57(4): 794-802

[13] Ng, K.K.C., et al., Randomized clinical trial of hepatic resection versus radiofrequency ablation for early - stage hepatocellular carcinoma[J].John Wiley \& Sons, Ltd: Chichester, UK. 2017.24(1):1775-1784

[14] Rich, N.E., N.D. Parikh and A.G. Singal, Hepatocellular Carcinoma and Liver Transplantation: Changing Patterns and Practices[J].Current Treatment Options in Gastroenterology, 2017. 15(2): 296-304

[15] Orcutt, S.T. and D.A. Anaya, Liver Resection and Surgical Strategies for Management of Primary Liver Cancer[J].Cancer Control, 2018. 25(1): 1-15

[16] Hsiao, C., et al., Surgical resection versus radiofrequency ablation for Barcelona Clinic Liver Cancer very early stage hepatocellular carcinoma: long-term results of a single-center study[J]. The American Journal of Surgery, 2020

[17] Li, X., et al.,Laparoscopic hepatectomy versus radiofrequency ablation for hepatocellular carcinoma: a systematic review and meta-analysis[J].Cancer Management and Research,2019. 11: $5711-5724$

[18] Jiang, Y., et al., Efficacy of Hepatic Resection vs. Radiofrequency Ablation for Patients With Very-Early-Stage or Early-Stage Hepatocellular Carcinoma: A PopulationBased Study With Stratification by Age and Tumor Size[J]. Frontiers in Oncology, 2019. 9:113

[19] Huang, Y., et al., Comparison of Radiofrequency Ablation and Hepatic Resection for the Treatment of Hepatocellular Carcinoma $2 \mathrm{~cm}$ or Less[J].Journal of Vascular and Interventional Radiology, 2018. 29(9): 1218-1225

[20] Ye, Q., et al., Safety and Efficacy of Radiofrequency Ablation for Solitary Hepatocellular Carcinoma $(3-5 \mathrm{~cm})$ : a Propensity Score Matching Cohort Study[J].Journal of Gastrointestinal Surgery, 2019. 23(8): 1549-1558 
[21] Casadei Gardini, A., et al., Radiofrequency Ablation of hepatocellular carcinoma: a meta-analysis of overall survival and recurrence-free survival[J].OncoTargets and Therapy,2018. 11: 6555-6567

[22] Pan, Y., et al., Radiofrequency ablation versus laparoscopic hepatectomy for hepatocellular carcinoma: A real world single center study[J].European Journal of Surgical Oncology, 2020. 46(4): 548-559

[23] Zheng, L., et al., Comparative Effectiveness of Radiofrequency Ablation vs. Surgical Resection for Patients With Solitary Hepatocellular Carcinoma Smaller Than 5 cm[J]. Frontiers in Oncology, 2020. 10:339 